\title{
Efectos clínicos de la corrección del valor de la fracción de expulsión del ventrículo izquierdo en pacientes con insuficiencia valvular mitral
}

\author{
Clinical impact of the correction of the value of left ventricular ejection fraction in \\ patients with mitral valve regurgitation
}

\author{
Hugo Villarroel-Ábrego ${ }^{1 *}$, Raúl Garillo², Hilda Peralta-Rosado ${ }^{3}$, Gustavo A. Lanosa $^{4}$, Carlos A. Perona ${ }^{5}$, \\ Gerardo de la Rosa-Veras ${ }^{6}$, Nilvia Castillo y Elaine Núñez ${ }^{7}$
}

\begin{abstract}
'Servicio de Cardiología, Fellow SIAC y SISIAC, Facultad de Medicina, Universidad Salvadoreña "Alberto Masferrer" (USAM), Hospital de Diagnóstico Escalón, San Salvador, El Salvador; ${ }^{2}$ Servicio de Cardiología, Facultad de Ciencias Médicas, Pontificia Universidad Católica Argentina, Buenos Aires, República Argentina; ${ }^{3}$ Servicio de Cardiología, FACC; Clínica de Mérida-Electrodiagnóstico del Sureste y del Hospital General Dr. Agustín O'Horan, Mérida, Yucatán, México; ${ }^{4}$ Servicio de Cardiología, Hospital Donación Francisco Santojanni y Fundación Científica del Sur, Buenos Aires, Argentina; ${ }^{5}$ Servicio de Cardiología, Hospital Donación Francisco Santojanni, Buenos Aires, Argentina; ${ }^{6}$ Laboratorio de Ecocardiografía del Centro Cardiovascular CEDIMAT, Santo Domingo, República Dominicana; ${ }^{7}$ Servicio de Cardiología, Corazones Unidos, CEDISA y Centro Médico Dominicano, Santo Domingo, República Dominicana
\end{abstract}

\begin{abstract}
Resumen
Introducción y objetivos: La insuficiencia valvular mitral provoca el vaciado simultáneo hacia la aorta y la aurícula izquierda durante la sístole ventricular, lo que produce una disminución del volumen hacia la circulación sistémica. En este estudio se busca obtener un dato preciso del porcentaje de volumen expulsado en sentido anterógrado en pacientes con insuficiencia mitral. Métodos: Se aplica una fórmula ecocardiográfica de "corrección" de la fracción de expulsión del ventrículo izquierdo (FEVI) en 114 pacientes con insuficiencia mitral, con base en la medición de la fracción regurgitante. Resultados: La corrección de la FEVI demostró que el $44.7 \%$ de los casos $(n=51)$ debe reclasificarse en cuanto a la calidad de su función sistólica ventricular izquierda. De 79 sujetos con FEVI normal ( $\geq 50 \%$ ) sólo se mantuvieron 32 en la misma categoría; en el grupo con FEVI moderadamente reducida (intervalo intermedio, 40-49.9\%) se pasó de 6 a 23 casos y, en aquéllos con FEVI reducida (< 40\%), el grupo aumentó de 29 a 59; el subgrupo de pacientes con $\mathrm{FEVI}<30 \%$ se incrementó de 21 a 41 sujetos. Conclusiones: Puesto que en la mayoría de las guías de tratamiento la FEVI se usa para estratificar riesgos e indicaciones terapéuticas, los autores creen que la ponderación de la insuficiencia mitral puede incrementar la precisión del tratamiento y la posibilidad de incluir a pacientes que no están considerados en esos tratamientos en el momento actual.
\end{abstract}

Palabras clave: Fracción de expulsión del ventrículo izquierdo. Insuficiencia mitral. Fracción regurgitante.

\begin{abstract}
Introduction and objectives: Mitral valve regurgitation causes simultaneous emptying to the aorta and left atrium during ventricular systole, generating a decrease in volume supply to the systemic circulation. In this study we seek to obtain an accurate
\end{abstract}

\section{Correspondencia:}

*Hugo Villarroel-Ábrego

E-mail: h_villarroel@yahoo.com

1405-9940/๑ 2020 Instituto Nacional de Cardiología Ignacio Chávez. Publicado por Permanyer. Este es un artículo open access bajo la licencia CC BY-NC-ND (http://creativecommons.org/licenses/by-nc-nd/4.0/). 
data on the percentage of volume expelled in the anterograde direction in patients with mitral regurgitation. Methods: An echocardiographic formula for "correction" of the left ventricular ejection fraction (LVEF) was applied in 114 patients with mitral regurgitation, based on the measurement of the regurgitant fraction. Results: Correction of the LVEF showed that $44.7 \%$ of cases $(n=51)$ should be reclassified in terms of the quality of their left ventricular systolic function. Of 79 subjects with normal LVEF $(\geq 50 \%$ ) only 32 remained in the same category; in the group with moderately reduced LVEF (medium range, 40-49.9\%) it went from 6 to 23 cases and, in those with reduced LVEF (<40\%), the group increased from 29 to 59; the subgroup of patients with LVEF $<30 \%$ increased from 21 to 41 subjects. Conclusions: Given that in most treatment guidelines LVEF is used to stratify risks and therapeutic indications, the authors believe that the weighting of mitral regurgitation can increase the accuracy of treatment, and the possibility of including patients who, at this current moment, are not considered for these therapies.

Key words: Left ventricular ejection fraction. Mitral regurgitation. Regurgitant fraction.

\section{Introducción}

La insuficiencia valvular mitral sugiere una disminución del flujo anterógrado que suministra el ventrículo izquierdo, lo cual genera una respuesta contráctil adaptativa para compensar, en cierta medida, la reducción del volumen sistólico; esto causa por lo general una mayor fracción de expulsión del ventrículo izquierdo $(\mathrm{FEVI})^{1}$. Tal aumento de la FEVI es característico de los ventrículos con buena reserva contráctil, de tal modo que puede considerarse una disfunción ventricular si un paciente con insuficiencia mitral moderada o grave registra valores de FEVI cercanos a los de referencia. En consecuencia, el valor aislado de la FEVI no permite que ciertos pacientes con insuficiencia cardíaca puedan clasificarse y tratarse de forma correcta de acuerdo con las guías, cuyas recomendaciones se ajustan muchas veces a la condición de la misma FEVI.

Se ha demostrado que en individuos con insuficiencia cardíaca las regurgitaciones mitrales son muy frecuentes $^{2}$, por lo que un porcentaje considerable de pacientes podría tener disfunción sistólica no detectada con valores de FEVI en apariencia normales o preservados, lo que impide establecer el diagnóstico.

El objetivo de esta investigación es obtener un porcentaje preciso del volumen expulsado en sentido anterógrado en pacientes con insuficiencia mitral, por medio de una fórmula que, a partir de la fracción regurgitante, permita "corregir" el valor de la FEVI y demostrar la existencia de una disfunción ventricular no sospechada.

\section{Métodos}

Para calcular la FEVI "corregida" $\left(\mathrm{FEVI}_{\mathrm{C}}\right)$ por ecocardiografía se requieren tres datos:

- FEVI

- $V L_{R}$ mitral

- Fracción regurgitante de la insuficiencia mitral (FR)
Para calcular el $\mathrm{VL}_{\mathrm{R}}$ pueden emplearse dos métodos:

- Medición del área del hemisferio de isovelocidad proximal (PISA)

- Ecuación de continuidad

Si se emplea el método PISA entonces:

$$
\mathrm{VL}_{\mathrm{R}}=\left[\mathrm{EROA}\left(\mathrm{cm}^{2}\right) \times \mathrm{ITV} \mathrm{IM}(\mathrm{cm})\right]
$$

En esta fórmula, EROA es el área del orificio regurgitante y la ITV IM la integral de la curva tiempo-velocidad del chorro del flujo de la insuficiencia mitral, obtenida con Doppler de onda continua.

Si se utiliza la ecuación de continuidad deben calcularse y restarse entre sí los volúmenes de flujo transmitral $\left(V_{V_{M}}\right)$ y flujo transaórtico $\left(\mathrm{VL}_{\mathrm{A}_{0}}\right)$ :

$\mathrm{VL}_{\mathrm{VM}}=$ área del anillo mitral $\mathrm{x}$ ITV del anillo mitral

$\mathrm{VL}_{\mathrm{Ao}}=$ área del anillo aórtico $\mathrm{x}$ ITV del anillo aórtico

En estas fórmulas, ITV es la integral tiempo-velocidad. La fórmula del $V L_{R}$ es entonces:

$$
\mathrm{VL}_{\mathrm{R}}=\mathrm{VL}_{\mathrm{TM}}-\mathrm{VL}_{\mathrm{Ao}}
$$

Al calcular el $\mathrm{VL}_{\mathrm{R}}$ por cualquiera de ambos métodos se determina la fracción regurgitante (FR) de la insuficiencia mitral, es decir, el porcentaje de todo el volumen expulsado del ventrículo izquierdo que resulta regurgitado a la aurícula izquierda:

$$
\mathrm{FR}=\left(\mathrm{VL}_{\mathrm{R}} / \mathrm{VL}_{\mathrm{VM}}\right) \times 100
$$

La FEVI corregida $\left(\mathrm{FEVI}_{\text {corr }}\right)$ se calcula entonces de la siguiente manera:

$$
\mathrm{FEVI}_{\mathrm{C}}=(1-\mathrm{FR}) \mathrm{FEVI}
$$

Este estudio postula la necesidad de corregir la FEVI como procedimiento regular en la práctica ecocardiográfica. Se toma un valor de FEVI de 50\% como valor de corte para el análisis, dado que esa cifra es el valor de corte para definir disfunción ventricular según las guías vigentes ${ }^{3}$; la finalidad es detectar, entre otros objetivos, casos nuevos de FEVI en valores limítrofes 0 reducidos. Asimismo, se depura el conocimiento acerca de los procedimientos más adecuados para dicha corrección, de manera específica: 
- Estudiar la concordancia entre dos métodos diferentes (PISA y ecuación de continuidad) para calcular la FR.

- Verificar la correlación entre la derivada de presión/ derivada de tiempo (dP/dT) del chorro de insuficiencia mitral, tanto con la FEVI original como con la FEVIc.

- Determinar qué porcentaje de pacientes con insuficiencia mitral y función ventricular normal (FEVI $\geq$ $50 \%$ ) debe reclasificarse como casos de disfunción sistólica (FEVI < 50\%).

- Establecer el porcentaje de pacientes con insuficiencia mitral y función ventricular moderadamente reducida (FEVI de 40-49.9\%) que tiene una FEVI corregida $<40 \%$, y por tanto reclasificarlos como enfermos con insuficiencia cardíaca con FEVI reducida.

- Precisar el porcentaje de pacientes con insuficiencia mitral que tienen FEVI corregida $<30 \%$ (disfunción sistólica grave), lo cual puede llevar en casos seleccionados a indicar el implante de desfibriladores o resincronizadores, y que antes no eran elegibles por el simple criterio de la $\mathrm{FEVI}$, según las guías vigentes ${ }^{3}$.

Entre el 1 de mayo de 2017 y el 1 de noviembre de 2018 se incluyó a 114 pacientes con insuficiencia mitral en los laboratorios de ecocardiografía involucrados, en El Salvador, México, República Dominicana y Argentina. Se obtuvo la autorización para la ejecución del estudio por parte de los comités de ética de todos los hospitales y clínicas participantes.

\section{Inclusión}

\section{Criterios de inclusión}

- Ritmo regular con frecuencia cardíaca de 50 a $100 / \mathrm{min}$.

- Imágenes bidimensionales o tridimensionales de alta calidad de los planos valvulares y ventrículo izquierdos.

- Registro Doppler pulsado nítido de los flujos transmitral y transaórtico a nivel de los planos anulares.

- Hemisferio de isovelocidad proximal cuantificable.

- Chorro de insuficiencia mitral susceptible de medición con el Doppler de onda pulsada.

\section{Criterios de exclusión}

- Estenosis mitral.

- Ritmo diferente del sinusal durante el ecocardiograma.

- Insuficiencia aórtica más allá de la regurgitación mínima, con vena contracta $>3 \mathrm{~mm}, 0$ EROA (área de orificio regurgitante) $>0.1 \mathrm{~cm}^{2}$.
- Cortocircuitos intracardíacos o extracardíacos (se incluye al paciente si tan sólo se trata de agujero oval permeable).

- Pacientes con dispositivos: marcapasos, cardiodesfibriladores implantables o resincronizadores, dispositivos de asistencia ventricular. Se permitió incluir algunos casos en los que el paciente implantado se encontraba en ritmo nativo (sin ninguna estimulación) durante las mediciones.

Se registraron las siguientes variables clínicas/antropométricas: género, edad, peso, talla, índice de masa corporal (IMC), área de superficie corporal (ASC), presión arterial (sistólica, diastólica y media). Se revisaron los electrocardiogramas para descartar criterios de exclusión y se registró la presencia de hipertensión arterial, diabetes mellitus, cardiopatía isquémica, nefropatía crónica o enfermedad pulmonar obstructiva crónica. También se confirmó si existía una indicación para tratamiento de resincronización cardíaca o implante de desfibrilador automático.

\section{Procedimientos ecocardiográficos}

Los estudios ecocardiográficos se realizaron con los equipos Siemens Acuson SC 2000, Acuson X700, GE Vivid 7, GE Vivid 3 PRO, GE Vivid S6, ESAOTE My Lab 50 y Phillips Epic 7c.

Se tomaron las siguientes mediciones:

1. FEVI según los procedimientos ampliamente detallados en las publicaciones médicas ${ }^{4}$ : volúmenes ventriculares izquierdos al final de la diástole (VfDVI) y la sístole (VfSVI), volumen latido (VL); FEVI, mediante planimetría de área longitud en dos planos, Simpson o ecocardiografía tridimensional.

2. Corrección de la FEVI.

a. Medición del volumen latido regurgitante a la aurícula izquierda $\left(\mathrm{VL}_{\mathrm{R}}\right)$. Esto puede realizarse de dos maneras:

- Método de medición del área del hemisferio de isovelocidad proximal (PISA) $)^{5-7}$

- Método basado en la ecuación de continuidad ${ }^{5,6}$

b.Cálculo de la fracción regurgitante (FR).

c. Aplicación de la fórmula de corrección de FEVI.

\section{Procedimientos específicos}

\section{MÉTOdo de ÁREA DE ISOVELOCIDAD PROXIMAL (PISA) PARA CUANTIFICAR LA INSUFICIENCIA MITRAL}

- Diámetro del anillo mitral en mesodiástole, cálculo del radio (diámetro/2) y área del anillo (radio al cuadrado multiplicado por 3.1416). 


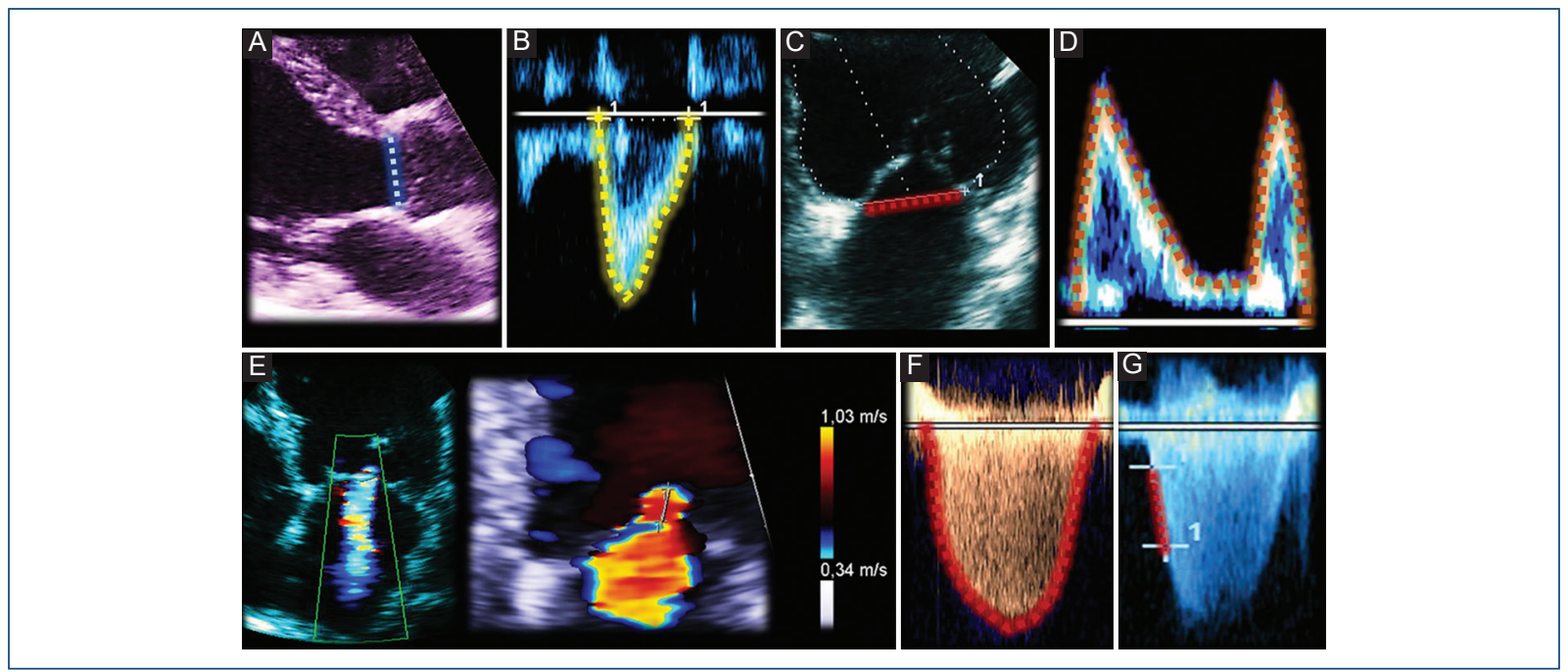

Figura 1. Procedimientos ecocardiográficos. A: Anillo aórtico en sístole (equivale al tracto de salida del ventrículo izquierdo). B: Integral tiempo-velocidad de flujo a nivel del anillo aórtico. C: Diámetro del anillo mitral en la mesodiástole. D: Integral tiempo-velocidad de flujo a nivel del anillo mitral. E: Chorro de insuficiencia mitral, ajuste de la velocidad de aliasing y medición del radio de isovelocidad proximal. F: Integral tiempo-velocidad del chorro de insuficiencia mitral. G: Medición de dP/dT.

- Integral tiempo-velocidad del flujo transmitral (ITV VM) a nivel del anillo.

- Radio y área de isovelocidad proximal (PISA) del chorro de insuficiencia mitral.

- Velocidad de aliasing (Nyquist).

- Integral tiempo-velocidad del chorro de insuficiencia mitral (ITV IM).

- Derivada de presión/tiempo (dP/dT) de la aceleración del chorro de insuficiencia mitral.

- Área del orificio regurgitante (EROA).

- Integral tiempo-velocidad del chorro de insuficiencia mitral (ITV IM).

-. Área del anillo mitral.

- Volumen latido transmitral $\left(\mathrm{VL}_{\mathrm{VM}}\right)$.

\section{MÉTOdO POR ECUACIÓN DE CONTINUIDAD}

- Área del tracto de salida del ventrículo izquierdo.

- Integral tiempo-velocidad del flujo aórtico (ITV Ao).

- Volumen latido aórtico $\left(\mathrm{VL}_{\mathrm{AO}_{0}}\right)$.

- Área del anillo mitral.

- Integral tiempo-velocidad del flujo diastólico mitral (ITV VMd) a nivel del anillo.

- Volumen latido mitral $\left(V L_{V M}\right)$ :

- Medición de la diferencia entre los volúmenes anterógrados de ambas válvulas (mitral menos aórtica) para disponer del dato de $\mathrm{VL}_{\mathrm{R}}$.

En la figura 1 se muestran imágenes representativas de los procedimientos de medición y cálculo descritos.
Se registraron los resultados por ambos métodos, además del promedio de $\mathrm{VL}_{\mathrm{R}}$, $\mathrm{FR}$ y las respectivas $\mathrm{FEVI}$ corregidas.

\section{Otras mediciones}

- Con la finalidad de disponer de más elementos de juicio para la valoración de la función sistólica se cuantificó también la derivada de presión/tiempo (dP/dT) de la aceleración del chorro de insuficiencia mitral.

- Se estudiará a un subgrupo de pacientes en el que pueda medirse el strain longitudinal global y se tabularán sus datos para fines de otra investigación en paralelo a cargo del mismo equipo.

Todos los resultados deben ser el promedio de al menos tres mediciones y registrarse de modo digital.

\section{Análisis estadístico}

El análisis estadístico incluyó:

- Método de Bland-Altman para valorar la concordancia entre los valores de fracción regurgitante obtenidos por métodos PISA y ecuación de continuidad.

- Estudio de correlación (Pearson) entre los valores de potencia ventricular (integral presión-tiempo, equivalente a fuerza-tiempo) usando el valor de $\mathrm{dP} / \mathrm{dt}$ de la aceleración del chorro de insuficiencia mitral, contra los valores de FEVI, corregida y sin corregir. 


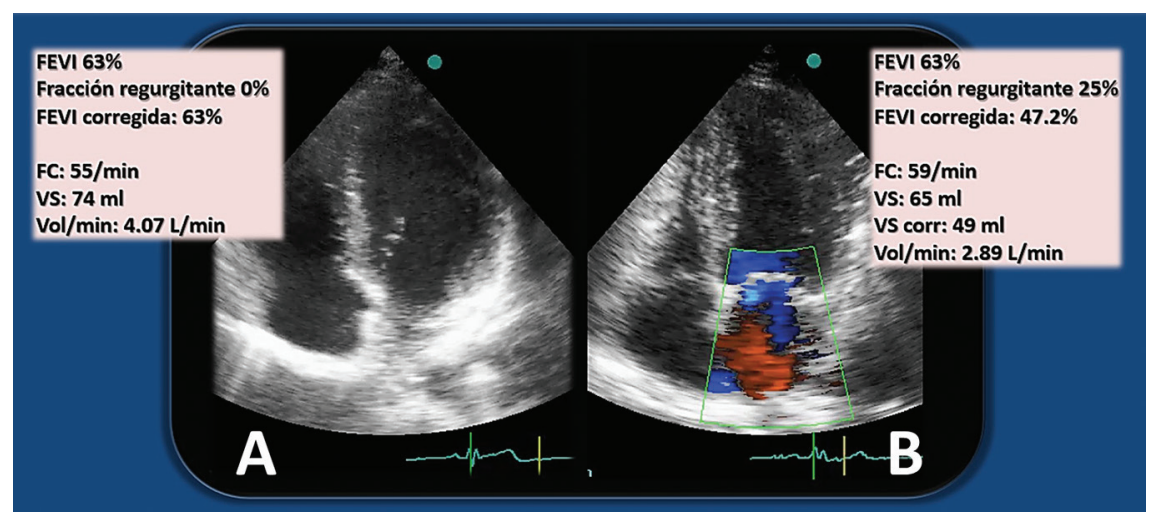

Figura 2. Efecto de la corrección de la insuficiencia mitral en la hemodinamia; ejemplos basados en ecocardiografía, vistas apicales de cuatro cámaras. A: Un paciente sin morbilidad ni insuficiencia mitral, con FEVI normal de $63 \%$. B: Paciente hipertenso con la misma FEVI, pero con insuficiencia mitral con fracción regurgitante (FR) del $25 \%$. Al aplicar la fórmula correctiva, la FEVI anterógrada desciende hasta 47.2\%; esto da lugar a que el volumen sistólico (VS) y el gasto cardíaco (GC) se vean también mermados.

Tabla 1. Distribución de pacientes de acuerdo con las características clínicas al momento de la inclusión

\begin{tabular}{|l|c|c|}
\hline Variable clínica & $\mathbf{n}$ & $\%$ \\
\hline Edad $>65$ años & 71 & 62.3 \\
\hline Hipertensión arterial & 58 & 54.7 \\
\hline Obesidad (IMC $\geq 30 \mathrm{~kg} / \mathrm{m}^{2}$ ) & 30 & 26.3 \\
\hline Diabetes mellitus & 22 & 20.7 \\
\hline Neumopatía crónica & 3 & 2.8 \\
\hline Nefropatía crónica & 14 & 13.2 \\
\hline Insuficiencia cardíaca & 44 & 38.6 \\
\hline FEVI $\geq 50 \%$ & 35 & 30.7 \\
\hline Enfermedad coronaria & 19 & 16.7 \\
\hline Cardiomiopatía no isquémica & 19 & 16.7 \\
\hline Cardiopatía chagásica & 4 & 3.8 \\
\hline Bloqueo avanzado de rama & 12 & 10.5 \\
\hline izquierda del haz de His & & \\
\hline
\end{tabular}

IMC: índice de masa corporal; FEVI: fracción de expulsión del ventrículo izquierdo.

- El porcentaje de pacientes estudiados con FEVI corregida se reclasificaría en términos del diagnóstico: FEVI normal $\left(\geq 50 \%{ }^{8}\right)$, FEVI intervalo medio (40\% - 49.9\%), FEVI reducida (<40\%), FEVI gravemente reducida ( $\leq 30 \%$ ).

- Valorar el porcentaje de pacientes estudiados con FEVI corregida que se convertiría en nuevos pacientes elegibles para TRC o implante de cardiodesfibrilador (o previamente excluidos por la FEVI sin corregir).

\section{Resultados}

Un total de 114 pacientes se incluyó en la muestra final. El sexo femenino $(n=60)$ predominó sobre el masculino $(n=54): 52.6$ vs. $47.4 \%$. La media de edad fue de $66.4 \pm 15.7$ años (intervalo, 18-94 años). Las variables clínicas se resumen en la tabla 1 .

La mayoría de los casos era de insuficiencia mitral leve $(\mathrm{FR}<30 \%)$ con 61 (53.5\%); 18.4\% de los casos $(n=21)$ se consideró de repercusión grave ( $F R>50 \%)$; los restantes $32(28.1 \%)$ tenían insuficiencia mitral moderada. En cuanto a las causas de las regurgitaciones, según la clasificación de Carpentier, se registraron 65 casos del tipo I (57\%), 11 de tipo II (movilidad excesiva, prolapso mitral, incluido un caso de síndrome de Marfan), ningún caso del tipo IIIA y 38 casos del tipo IIIB, por tethering del aparato subvalvular $(n=38)$ causados por dilatación ventricular izquierda.

El cálculo de la FR por ecuación de continuidad fue posible en todos los pacientes, excepto en $3(97.4 \%)$; el método PISA fue técnicamente satisfactorio en 89 pacientes $(78.9 \%)$ y la causa más frecuente de falta de aplicación fue la presencia de más de un chorro de regurgitación mitral, seguido de hemisferios con pobre definición. La concordancia entre los dos métodos de cálculo de la FR (que fue posible valorar en 87 casos, $76.3 \%$ de todos los estudios) fue muy alta y satisfactoria dado que se logró en el $97.7 \%$ de los ecocardiogramas, con un margen de error $<5 \%$ mediante el método de Bland-Altman (Fig. 2). Por esa razón se decidió promediar ambos valores cuando estuvieran 


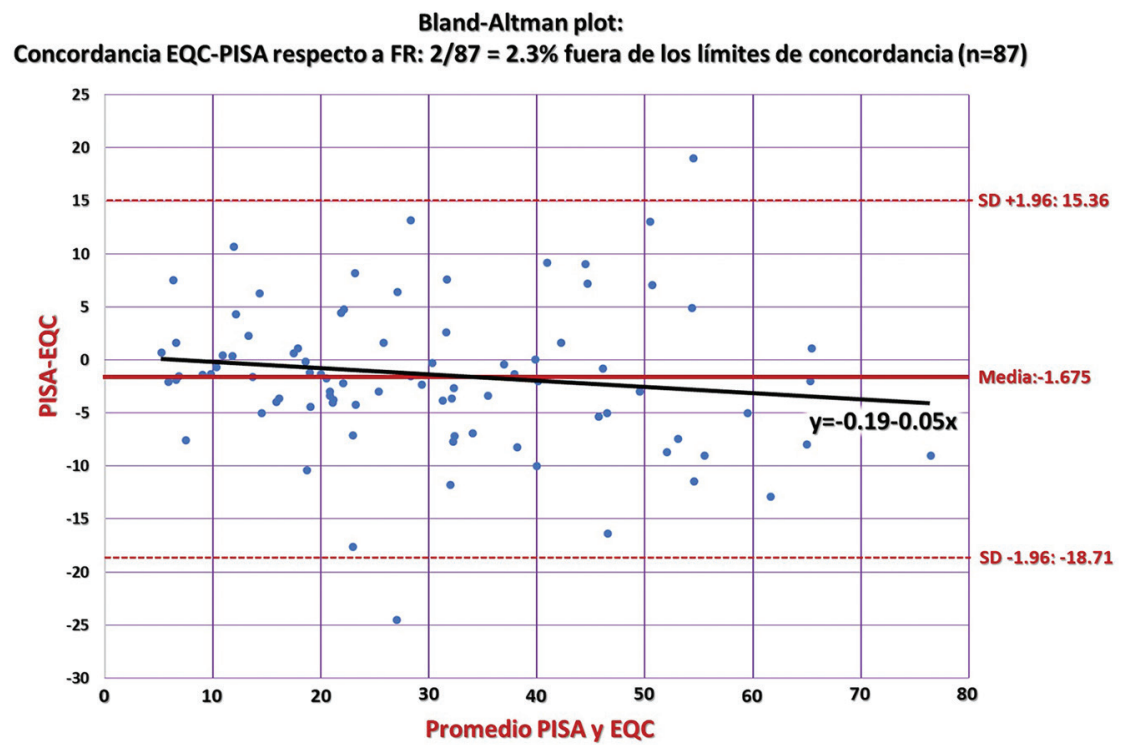

Figura 3. Gráfica de Bland-Altman para confirmar la concordancia entre métodos para cálculo de la fracción regurgitante de insuficiencia mitral. PISA: área de superficie de isovelocidad proximal; EQC: ecuación de continuidad.

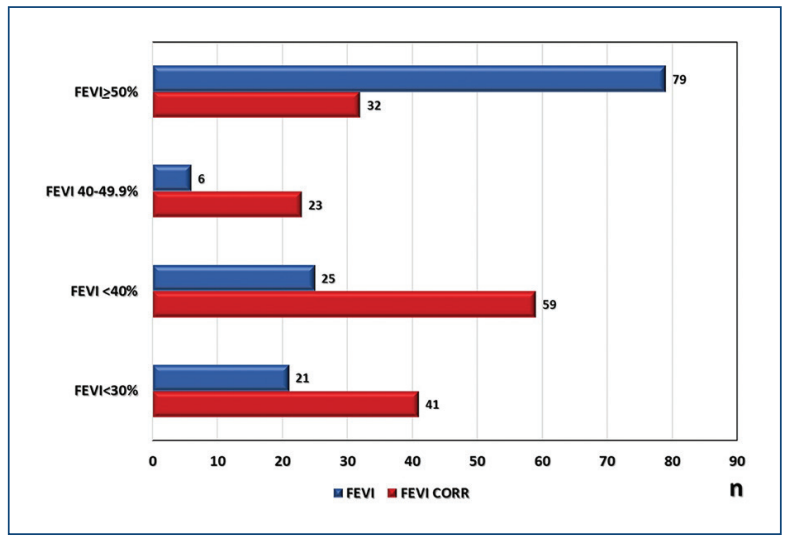

Figura 4. Clasificación de los pacientes con insuficiencia mitral en cuanto a la fracción de expulsión del ventrículo izquierdo (FEVI) antes (barras azules) y después de la corrección (barras rojas). $\mathrm{n}$ = número de casos.

disponibles, para el procesamiento ulterior de la información, es decir, el cálculo de la FEVI corregida.

Al corregirse las FEVI, una gran proporción de los pacientes debió reclasificarse en cuanto a la calidad de su función ventricular izquierda (51 de 114, 44.7\% de casos). De 79 sujetos con FEVI normal (> 50\%) sólo 32 se mantuvieron en la misma categoría; en el grupo con FEVI de 40 a $49.9 \%$, el grupo se incrementó de 6 a 23 pacientes; por su parte, en el de FEVI reducida
Tabla 2. Reclasificación de pacientes a diferentes categorías de FEVI de acuerdo con la gravedad de la insuficiencia mitral

\begin{tabular}{|l|c|c|c|}
\hline $\begin{array}{l}\text { Gravedad de la insuficiencia } \\
\text { mitral }\end{array}$ & $\mathbf{n}$ & $\begin{array}{c}\text { Casos } \\
\text { reclasificados }\end{array}$ & $\%$ \\
\hline Leve (FR $<30 \%)$ & 61 & 21 & 22.9 \\
\hline Moderada (FR $=30-49.9 \%)$ & 32 & 19 & 56.2 \\
\hline Grave (FR $>50 \%)$ & 21 & 11 & 52.4 \\
\hline $\begin{array}{l}\text { Todos los grados de } \\
\text { insuficiencia mitral }\end{array}$ & 114 & 51 & 44.7 \\
\hline FR: fracción regurgitante. & & & \\
\hline
\end{tabular}

$(<40 \%)$, los pacientes aumentaron de 29 a 59; en particular en aquéllos con $\mathrm{FEVI}<30 \%$, la cantidad de sujetos se incrementó de 21 a 41 (Fig. 3). Un ejemplo del efecto de la corrección de la FEVI sobre la hemodinamia se presenta en la figura 2 al comparar dos ventrículos con idéntica $\mathrm{FEVI}$, uno sin valvulopatía y otro con insuficiencia mitral.

Se realizó análisis ANOVA para muestras repetidas de las FEVI antes y después de la corrección y se identificó una diferencia estadísticamente muy significativa $(p<0.0001)$. Los casos en que hubo cambio de categoría de FEVI de acuerdo con la gravedad de la insuficiencia mitral se describen en la tabla 2. 


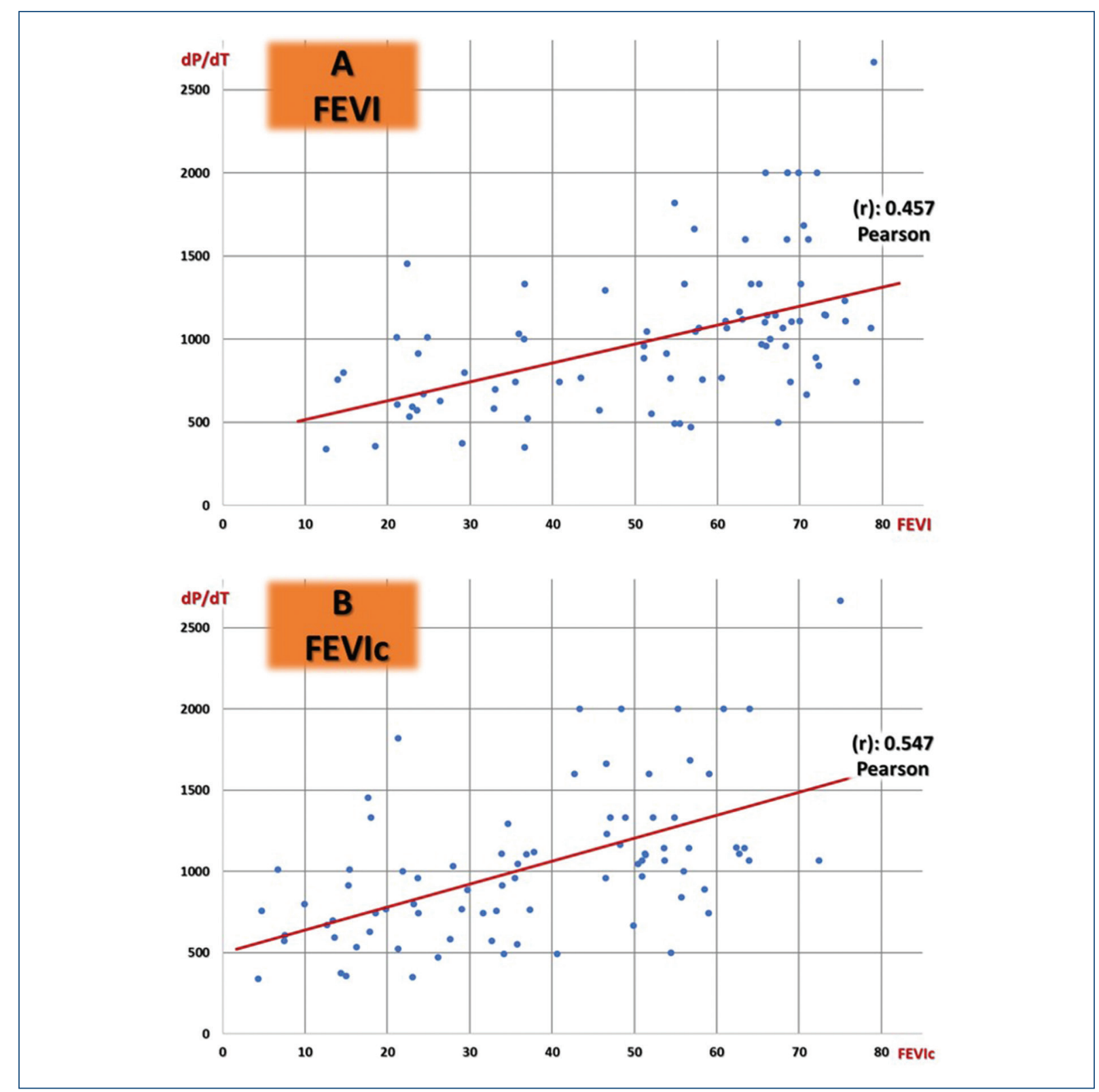

Figura 5. A: Correlación entre FEVI y dP/dT. B: FEVI corregida (FEVIc) y dP/dT mediante el coeficientes de Pearson.

Se ha planteado que es razonable determinar un valor de corte de $\mathrm{dP} / \mathrm{dT}$ de $1,000 \mathrm{mmHg} / \mathrm{s}$ para definir disfunción sistólica con este medio solo ${ }^{9}$. La correlación entre FEVI y dP/dT fue positiva pero débil, un aspecto ya descrito en las publicaciones médicas ${ }^{9}$, aunque al correlacionar FEVI corregida con $\mathrm{dP} / \mathrm{dT}$ el coeficiente de Pearson mejora discretamente, desde 0.457 hasta $0.547(p=0.6335$, sin diferencia significativa; Fig. 5).

Si se toman en cuenta las guías vigentes para el implante de cardiodesfibriladores automáticos y de tratamiento de resincronización cardíaca, se determinó el número de los 114 pacientes que podían ser elegibles para el tratamiento con dispositivos según fuera su FEVI corregida. Un total de 13 pacientes (11.4\%) calificaría, hipotéticamente, al considerar su clínica y el valor de su FEVI corregida; 10 de estos casos tenían una FR entre 30 y $50 \%$ y 3 una $F R>50 \%$; ningún paciente con insuficiencia mitral leve calificó para dispositivos. En 11 casos se satisfarían los criterios para implante de DAl y dos de los 12 pacientes con bloqueo avanzado de rama izquierda requerirían DAI + TRC. 


\section{Discusión}

Esta comunicación propone una fórmula correctiva relativamente sencilla para valorar el volumen expulsado a la circulación general en enfermos con IM significativa, más allá del valor de FEVI que se obtiene de los estudios efectuados. Implica a su vez que muchos pacientes deben reclasificarse en cuanto a la calidad de su función ventricular izquierda, al menos desde el criterio del valor de la FEVI. Este estudio pertenece al ámbito del "mundo real" ya que es una muestra final de pacientes consecutivos, no preseleccionados, con diferentes afecciones subyacentes, típicos consultantes de cardiología general; esto permite pensar en que los resultados pueden también extrapolarse a poblaciones mucho más grandes.

La clave para una corrección de la FEVI es un cálculo de calidad de la fracción regurgitante de la insuficiencia mitral. Aunque hay dos métodos ecocardiográficos validados, existen notorias limitantes que afectan a ambos, en especial al PISA, en cuanto a la medición del EROA. Sin embargo, en este estudio se ha demostrado un alto valor de concordancia entre PISA y ecuación de continuidad, a tal grado que si un método no es fiable el otro puede emplearse con el suficiente grado de confianza.

No sorprende la muy modesta correlación entre FEVI y dP/dT del chorro de insuficiencia mitral. Ambos parámetros se ven muy influidos por las condiciones de carga y por eso ninguno de ellos es adecuado para valorar la reserva inotrópica. Aunque hay una discreta mejoría de la correlación al corregir la FEVI, los autores creen que el valor de $\mathrm{dP} / \mathrm{dT}$ podría ser más útil como valoración secuencial de pacientes y menos como método de tamizaje para diagnóstico de disfunción ventricular.

Estos resultados podrían tener repercusiones importantes en el ámbito clínico porque podrían explicar por qué hay a menudo una discordancia entre síntomas y FEVI o entre FEVI y otros parámetros basados en imágenes, como la ecocardiografía de $\operatorname{strain}^{10}$. Por otra parte, los diagnósticos basados en valores de corte de FEVI, como catalogar a un paciente como afectado de insuficiencia cardíaca con función ventricular preservada o reducida, podrían y quizás deberían modificarse a la luz de estos nuevos valores corregidos de FEVI. Más aún, las decisiones terapéuticas fundamentadas en la FEVI ${ }^{11}$ (uso de ciertos fármacos, implante de TRC o indicación de DAl) podrían tomarse de manera más temprana y oportuna, como es el caso de los 11 pacientes aptos para implante de dispositivos.

\section{Conclusión}

La corrección de la FEVI con la fórmula propuesta deber ser un procedimiento habitual en cardiología; la fórmula misma podría incorporarse a los paquetes de cálculo de los equipos de ecografía. Se necesita más investigación para los casos excluidos aquí, como los pacientes con estimulación artificial y aquellos que se hallan bajo ritmo no sinusal.

¿Qué se sabe en realidad de este tema?

- La insuficiencia mitral es frecuente en pacientes con insuficiencia cardíaca.

- Muchas decisiones terapéuticas dependen de una medición óptima de la FEVI.

- La FEVI valora de modo indirecto la función sistólica pero rara vez se calcula tras considerar el valor real del volumen sistólico anterógrado si hay insuficiencia mitral.

¿Qué nuevos aportes existen?

- La corrección de la FEVI puede efectuarse de modo rápido y eficiente con el empleo de una sencilla fórmula: (1-FR) x FEVI.

- La FEVI corregida permite reclasificar a los pacientes afectados de insuficiencia mitral con respecto a la calidad de su función ventricular izquierda.

- Al reclasificar la función ventricular izquierda con base en un valor corregido de FEVI (<30 o 35\%), se abren opciones para considerar ciertos tratamientos como resincronización o implante de cardiodesfibriladores.

\section{Financiamiento}

Ninguno de los autores ha recibido financiamiento para la ejecución del presente estudio.

\section{Conflicto de intereses}

Ninguno de los autores declara conflictos de interés.

\section{Responsabilidades éticas}

Protección de personas y animales. Los autores declaran que para esta investigación no se han realizado experimentos en seres humanos ni en animales.

Confidencialidad de los datos. Los autores declaran que han seguido los protocolos de su centro de trabajo sobre la publicación de datos de pacientes. 
Derecho a la privacidad y consentimiento informado. Los autores declaran que en este artículo no aparecen datos de pacientes.

\section{Bibliografía}

1. Starling MR, Kirsh MM, Montgomery DG. Impaired left ventricular contractile function in patients with long-term mitral regurgitation and normal ejection fraction. JACC. 1993;22(1):239-250.

2. Varadarajan $P$, Sharma $S$, Heywood T. High prevalence of clinically silent severe mitral regurgitation in patients with heart failure: role for echocardiography. J Am Soc Echocardiogr. 2006:19:1458-1461.

3. 2016 ESC Guidelines for the Diagnosis and Treatment of Acute and Chronic Heart Failure. The Task Force for the Diagnosis and Treatment of Acute and Chronic Heart Failure of the European Society of Cardiology (ESC). Developed with the special contribution of the Heart Failure Association (HFA) of the ESC. European Journal of Heart Failure. 2016. doi:10.1002/ejhf.592.

4. Lang RM, Bierig M, Devereux RB. Recomendaciones para la cuantificación de las cavidades: informe del comité de guías y estándares de la sociedad americana de ecocardiografía y del grupo redactor de la cuan- tificación de las cavidades, desarrollado conjuntamente con la Asociación Europea de Ecocardiografía, rama de la Sociedad Europea de Cardiología. J Am Soc Echocardiogr. 2005;18:1440-1463.

5. García-Fernández MA, Gómez de Diego JJ. Cuantificación en Ecocardiografía. Momento Médico, Srl. Salerno, Italia, 2011:107-109.

6. Zoghbi WA, Adams D, Bonow RO. Recommendations for noninvasive evaluation of native valvular regurgitation: a report from the American Society of Echocardiography developed in collaboration with the society for cardiovascular magnetic resonance. Journal of the American Society of Echocardiography. 2017:303-371.

7. Enciso R, Summerson C, Aguilar JA. Cuantificación de la insuficiencia mitral con el método del flujo convergente proximal por eco Doppler-color. Revista Mexicana de Cardiología. 1997;8(2):52-60.

8. Wood PW, Choy JB, Nanda NC, Becher H. Left ventricular ejection fraction and volumes: it depends on the imaging method. Echocardiography. 2014;31:87-100.

9. Álvarez-López M, Alcalá-López JE, Baún-Mellado O. Utilidad del índice Doppler dP/dt en la evaluación de la disfunción sistólica ventricular izquierda. Revista Española de Cardiología. 1997;50(2).

10. Villarroel-Ábrego H, Garillo R, González-Suero JC. Correlación entre Fracción de expulsión del ventrículo izquierdo y strain longitudinal global en pacientes con hipertensión arterial. Insuf Card. 2018;13(2):57-66.

11. Villarroel-Ábrego H, Garillo R, Peralta-Rosado H, Núñez-Ayala E, González-Suero JC. Fórmula para corregir la fracción de expulsión del ventrículo izquierdo en pacientes con insuficiencia valvular mitral. Rev Argent Cardiol. 2019;87:229-233. http://dx.doi.org/10.7775/rac.es.v87.i3.15163. 